\title{
Associated comorbidities with obesity in pregnancy and its feto- maternal outcome
}

\author{
Rupam S. Jain ${ }^{1 *}$, Sneha D. Shirodkar ${ }^{2}$
}

\begin{abstract}
${ }^{1}$ Department of Obstetrics and Gynecology, LTMMC and LTMGH, Sion, Mumbai, Maharashtra, India
${ }^{2}$ Department of Obstetrics and Gynecology, TNMC and BYL Nair Hospital, Sion, Mumbai, Maharashtra, India
\end{abstract}

Received: 05 May 2019

Accepted: 11 June 2019

\author{
*Correspondence: \\ Dr. Rupam S. Jain, \\ E-mail: rupamjain.91@gmail.com
}

Copyright: (C) the author(s), publisher and licensee Medip Academy. This is an open-access article distributed under the terms of the Creative Commons Attribution Non-Commercial License, which permits unrestricted non-commercial use, distribution, and reproduction in any medium, provided the original work is properly cited.

\begin{abstract}
Background: Obesity is an evolving risk factor in the modern day - a rising global epidemic reported to cause many problems in the pregnant woman - causing ill effects to both the mother and the fetus. Managing the comorbidities associated with obesity is a challenge in obstetric care.

Methods: This prospective observational cohort study was conducted at a tertiary care institute over a period of 2 years. 50 antenatal patients fulfilling the inclusion criteria were studied after taking informed consent. The various maternal, fetal and neonatal outcomes were noted to examine the magnitude and significance of the effect of BMI.

Results: Obese women have a higher prevalence of infertility and are considered a high-risk state because pregnancy is associated with a higher risk of pre-eclampsia, gestational diabetes mellitus, preterm labour, increased operative and caesarean deliveries, post-partum haemorrhage, post-partum infectious morbidities and a higher incidence of anesthetic and post-operative complications in these deliveries. Neonatal complications include congenital anomalies, fetal macrosomia leading to birth injuries, preterm births and still births. Technical difficulties are faced by the obstetricians while operating and by the anesthesiologists while induction.

Conclusions: Considering the adverse effects of obesity on pregnancy outcomes, pre-pregnancy counseling should be done for all overweight and obese females planning conception signifying the negative impact of obesity on maternal and fetal outcome.
\end{abstract}

Keywords: Body mass index, Diabetes mellitus, Gestational diabetes mellitus, Hypertension, Lower segment caesarean section, Pregnancy induced hypertension

\section{INTRODUCTION}

In the present-day scenario, obesity is a worldwide individual and public health issue because it contributes to the development of several chronic diseases thereby causing increased morbidity. ${ }^{1}$ The obesity epidemic affects all - including women of reproductive age group. ${ }^{2}$ Defined as pre-pregnancy BMI of $>=30$, obesity among women of reproductive age group is also reaching epidemic proportions.
Compared with normal-weight women, obese women have a higher prevalence of infertility. Once they conceive, they are considered a high-risk state because pregnancy is associated with a higher risk of preeclampsia, gestational diabetes mellitus, preterm labour, increased operative and caesarean deliveries, post-partum haemorrhage, post-partum infectious morbidities and a higher incidence of anaesthetic and post-operative complications in these deliveries. ${ }^{2}$ Neonatal complications include congenital anomalies, fetal 
macrosomia leading to birth injuries, preterm births and still births. Technical difficulties are faced by the obstetricians while operating and by the anaesthesiologists while induction. ${ }^{3}$

Obesity in pregnancy can also affect health later in life for both mother and child. For women, these risks include heart disease, diabetes mellitus and hypertension. Children have a risk of future obesity, diabetes and heart disease. $^{4}$

Taking into consideration the epidemic of obesity, with all its adverse long-term consequences, there is an increasing need to promote counselling before, during and after pregnancy on the role of diet and physical activity in reproductive health.

\section{METHODS}

This prospective observational cohort study was conducted at a tertiary care institute over a period of 2 years from October 2015 to September 2017 after obtaining ethical clearance for the study from the hospital's ethics committee. 63 antenatal patients fulfilling the inclusion criteria were studied after taking informed consent. 13 patients who were lost to follow up were excluded from the study.

All antenatal patients with BMI > 29.99 registered with or referred to Obstetrics and Gynaecology Department of the tertiary care institute were included in the study. BMI was calculated based on either the pre-pregnancy weight or weight in the $1^{\text {st }}$ trimester and patients were enrolled in the study in the $1^{\text {st }}$ trimester.

Patients not willing to participate in the study, patients with multifetal pregnancy and patients who failed to follow up were excluded from the study.

All antenatal patients at their first booking visit in the $1^{\text {st }}$ trimester underwent measurement of height (using a fixed meter scale) and weight (standard weighing machine). BMI of every patient was calculated as weight in kilograms divided by height in meters squared. Written informed consent was taken from all women willing to participate in the study.
Complete history, physical examination and relevant investigations of the patient were documented at registration. Periodic obstetric examination of each member of group was done as per standard antenatal protocol at every antenatal visit. The various maternal, fetal and neonatal outcomes were noted to examine the magnitude and significance of the effect of BMI.

\section{Statistical analysis}

In this study, all statistical analysis was performed by using 10.0 version of statistical software SPSS. Continuous variables were summarized by using summary statistics (number of observations, mean and standard deviation with range). Categorical values were estimated by using frequencies and percentages. In this study data associated with BMI and maternal age was estimated by Student $t$ test. For other parameters like pregnancy losses, conception, prevalence of infertility, comorbidities, duration of pregnancy, onset of labour, mode of delivery Chi Square test was used. All values were interpreted at $5 \%$ level of significance $(95 \%$ confidence interval).

\section{RESULTS}

In our study, the age of the cases ranged from 20 years 38 years with majority of the cases - $44 \%$ belonging to the age group of 25 years - 29 years (Table 1 ).

Table 1: Demographic data.

\begin{tabular}{|ll|}
\hline Age (years) & No. of cases \\
\hline $20-24$ & $9(18 \%)$ \\
\hline $25-29$ & $22(44 \%)$ \\
\hline $30-34$ & $12(24 \%)$ \\
\hline $35-39$ & $7(14 \%)$ \\
\hline
\end{tabular}

Table 2: BMI class distribution.

\begin{tabular}{|c|c|}
\hline No. of cases & 50 \\
\hline \multicolumn{2}{|c|}{ BMI class (\%) } \\
\hline Class 1 & $29(58 \%)$ \\
\hline Class $2 / 3$ & $21(42 \%)$ \\
\hline
\end{tabular}

$58 \%$ of the cases belonged to BMI class 1 and $42 \%$ of the cases belonged to BMI class 2/3 (Table 2).

Table 3: Association between BMI class and previous pregnancy losses.

\begin{tabular}{|c|c|c|c|c|c|}
\hline \multirow{3}{*}{ BMI class } & \multirow{3}{*}{$\begin{array}{l}\text { No. of cases } \\
(\mathbf{N}=\mathbf{5 0})\end{array}$} & \multicolumn{4}{|c|}{ Pregnancy losses } \\
\hline & & \multicolumn{2}{|c|}{ Abortions } & \multicolumn{2}{|c|}{ Still birth } \\
\hline & & No & $\%$ & No & $\%$ \\
\hline Class 1 & 29 & 12 & 41.4 & 03 & 10.3 \\
\hline Class $2 / 3$ & 21 & 06 & 28.6 & 01 & 04.8 \\
\hline
\end{tabular}


In our study, abortions occurred in $41.4 \%$ of the Class 1 BMI cases compared to $28.6 \%$ of the Class $2 / 3$ cases. $10.3 \%$ of the Class 1 BMI cases had still births compared to $4.8 \%$ of the Class $2 / 3$ BMI cases (Table 3 ). This was comparable to a study conducted by Lashen et al. ${ }^{5}$
Our study showed a decrease in the number of spontaneous conceptions with increase in BMI (Table 4). With increasing BMI, the prevalence of infertility increased from $3.4 \%$ in Class 1 BMI to $14.3 \%$ in Class $2 / 3$ BMI depicting a $2 \%$ lower fertility rate for every $\mathrm{kg} / \mathrm{m}^{2}$ increase in BMI.

Table 4: Association between BMI classes and conception.

\begin{tabular}{|c|c|c|c|c|c|}
\hline \multirow{3}{*}{ BMI class } & \multirow{3}{*}{$\begin{array}{l}\text { No. of cases } \\
(\mathbf{N}=\mathbf{5 0})\end{array}$} & \multicolumn{4}{|c|}{ Conception } \\
\hline & & \multicolumn{2}{|c|}{ Spontaneous } & \multicolumn{2}{|c|}{ Infertility } \\
\hline & & No & $\%$ & No & $\%$ \\
\hline Class 1 & 29 & 28 & 96.6 & 01 & 03.4 \\
\hline Class $2 / 3$ & 21 & 18 & 85.7 & 03 & 14.3 \\
\hline
\end{tabular}

Table 5: Association between BMI classes and complications in pregnancies.

\begin{tabular}{|c|c|c|c|c|c|}
\hline \multirow{3}{*}{ BMI Class } & \multirow{3}{*}{$\begin{array}{l}\text { No. of cases } \\
(\mathbf{N}=\mathbf{5 0})\end{array}$} & \multicolumn{4}{|c|}{ Pregnancies } \\
\hline & & \multicolumn{2}{|c|}{ Complicated } & \multicolumn{2}{|c|}{ Uneventful } \\
\hline & & No & $\%$ & No & $\%$ \\
\hline Class 1 & 29 & 21 & 72.4 & 08 & 27.6 \\
\hline Class 2/3 & 21 & 17 & 81.0 & 04 & 19.0 \\
\hline
\end{tabular}

Table 6: Association between BMI classes and comorbidities.

\begin{tabular}{|llcll|}
\hline \multirow{2}{*}{ Co-morbidities } & \multicolumn{2}{l|}{ BMI category } & \multicolumn{2}{l|}{ Class 2/3 $(\mathbf{N}=\mathbf{2 1})$} \\
\cline { 2 - 5 } & Class $\mathbf{1}(\mathbf{N}=\mathbf{2 9})$ & \multicolumn{2}{c|}{ No } & \% \\
\hline PIH & No & $\mathbf{\%}$ & 10 & 47.6 \\
\hline HTN & 17 & 58.6 & 01 & 04.8 \\
\hline GDM & 02 & 06.9 & 04 & 19.0 \\
\hline Type 2 DM & 03 & 10.3 & 02 & 09.5 \\
\hline Hypothyroidism & 04 & 13.8 & 06 & 28.6 \\
\hline
\end{tabular}

The rate of complications was $72.4 \%$ in Class 1 BMI cases compared to $81 \%$ in the Class $2 / 3$ BMI cases indicating an increase in the number of complicated pregnancies with an increase in BMI (Table 5).

Our study showed a positive co-relation of complications and obesity in pregnancy (Table 5).
Our study showed that PIH was present in $58.6 \%$ of Class $1 \mathrm{BMI}$ cases and $47.6 \%$ of Class $2 / 3 \mathrm{BMI}$ cases (Table 6) which was similar to a study conducted in Karachi by Asim et al, demonstrating PIH in $41 \%$ of obese pregnant women. ${ }^{6}$ Chronic hypertension was present in $6.9 \%$ of Class 1 BMI cases and $4.8 \%$ of Class $2 / 3$ BMI cases (Table 6).

Table 7: Association between BMI classes and duration of pregnancy.

\begin{tabular}{|c|c|c|c|c|}
\hline \multirow{3}{*}{ Duration of pregnancy } & \multicolumn{4}{|c|}{ BMI class } \\
\hline & \multicolumn{2}{|c|}{ Class $1(N=26)$} & \multicolumn{2}{|c|}{ Class $2 / 3(N=21)$} \\
\hline & No & $\%$ & No & $\%$ \\
\hline Preterm & 07 & 26.9 & 02 & 09.5 \\
\hline Postdatism & 07 & 26.9 & 04 & 19.1 \\
\hline
\end{tabular}

Our study showed that GDM was present in $10.3 \%$ of Class 1 BMI cases and 19\% of Class 2/3 BMI cases
(Table 6). A similar study by Zachary et al showed that GDM was present in $9.7 \%$ of Class 1 and $18.7 \%$ of Class 
2/3 BMI obese pregnant women. $13.8 \%$ of Class 1 BMI cases and $9.5 \%$ of Class 2/3 BMI cases were already suffering from Type 2 diabetes mellitus when recruited in the study (Table 6).

Our study showed the association of hypothyroidism in $6.9 \%$ of Class 1 BMI cases and a significantly higher $28.6 \%$ of Class 2/3 BMI cases (Table 6). A study conducted by Cheng et al in 2015 showed $11.11 \%$ prevalence of hypothyroidism in obese pregnant women. ${ }^{7}$

Our study showed an overall $22 \%$ incidence of postdatism with an incidence of $26.9 \%$ in Class 1 BMI cases and $19.1 \%$ in Class $2 / 3$ BMI cases (Table 7). Halloran et al 8 published a study which showed a similar incidence of postdatism $(16.45 \%)$ in obese pregnant women.

Table 8: Association between BMI classes and onset of labour.

\begin{tabular}{|c|c|c|c|c|}
\hline \multirow{3}{*}{ Onset of labour } & \multicolumn{4}{|c|}{ BMI class } \\
\hline & \multicolumn{2}{|c|}{ Class $1(N=26)$} & \multicolumn{2}{|c|}{ Class $2 / 3(N=21)$} \\
\hline & No & $\%$ & No & $\%$ \\
\hline Elective LSCS & 08 & 30.8 & 07 & 33.3 \\
\hline Induced & 11 & 42.3 & 06 & 28.6 \\
\hline Spontaneous & 07 & 26.9 & 08 & 38.1 \\
\hline
\end{tabular}

Table 9: Association between BMI classes and mode of delivery.

\begin{tabular}{|lllll|}
\hline \multirow{2}{*}{ Mode of delivery } & \multicolumn{3}{l}{ BMI class } & \multicolumn{3}{l|}{ Class 2/3 $(\mathbf{N}=\mathbf{2 1})$} \\
\hline & Class $\mathbf{1}(\mathbf{N}=\mathbf{2 6})$ & \multicolumn{2}{l|}{ No } & \% \\
\hline Normal vaginal & No & $\mathbf{\%}$ & 04 & 19.0 \\
\hline Operative & 10 & 38.5 & 01 & 04.8 \\
\hline LSCS & 01 & 03.8 & 16 & 76.2 \\
\hline
\end{tabular}

Our study showed a $26.9 \%$ incidence of preterm labour in Class 1 BMI cases (Table 7) in contrast to $5.54 \%$ incidence shown by a study conducted by Cnattingius et al, For class 2/3 BMI, the incidence of preterm labour was $9.5 \%$ in our study (Table 7) which was comparable to $6.64 \%$ in the study by Cnattingius et al. ${ }^{9}$

Our study showed that $26.9 \%$ of the Class 1 BMI cases and $38.1 \%$ of the Class $2 / 3$ BMI cases had spontaneous onset of labour and $42.3 \%$ of the Class 1 BMI cases and
$28.6 \%$ of the Class 2/3 BMI cases required induction of labour (Table 8 ) in view of either medical co-morbidities associated with obesity in pregnancy like PIH and GDM or postdatism. This was comparable to another study 10 which showed that $30.4 \%$ of Class $1 \mathrm{BMI}$ and $32.5 \%$ of Class 2/3 BMI required induction.

Rest of the cases i.e. $30.8 \%$ of Class 1 BMI cases and a slightly higher - $33.3 \%$ of Class $2 / 3$ BMI cases were planned for elective LSCS in view of obstetric indications (Table 8).

Table 10: Association between BMI classes and intra-partum complications.

\begin{tabular}{|c|c|c|c|c|c|c|}
\hline \multirow{3}{*}{ Intra- partum complications } & \multicolumn{6}{|c|}{ BMI category } \\
\hline & \multirow{2}{*}{$\mathbf{N}$} & \multicolumn{2}{|c|}{ Class 1} & \multirow[t]{2}{*}{$\mathbf{N}$} & \multicolumn{2}{|c|}{ Class $2 / 3$} \\
\hline & & No & $\%$ & & No & $\%$ \\
\hline Failure of induction & 11 & 04 & 36.4 & 06 & 03 & 50.0 \\
\hline Non - progress of labour & 14 & 03 & 21.4 & 11 & 04 & 36.4 \\
\hline VBAC failure & 05 & 03 & 60.0 & 01 & 01 & 100.0 \\
\hline Prolonged labour & 14 & 01 & 07.1 & 11 & 01 & 09.1 \\
\hline Shoulder dystocia & 11 & 02 & 18.2 & - & - & - \\
\hline Post-partum haemorrhage & 26 & 03 & 11.5 & 21 & 05 & 23.8 \\
\hline
\end{tabular}

Our study showed a declining trend of successful vaginal delivery with increase in BMI - 38.5\% in Class 1 BMI cases and $19 \%$ in Class 2/3 BMI cases (Table 9).
Our study showed a similar rate of operative vaginal delivery in both the classes (Table 9). The retrospective study in Missouri by Caughey et al carried out on obese 
pregnant women showed similar results $-5.9 \%$ in Class 1 BMI and 5.5\% in Class 2/3 BMI. ${ }^{11}$

Our study showed a high rate of caesarean sections in obese pregnant women (Table 9). The retrospective study by Caughey et al, showed lower rates of caesarean section in both the classes compared to our study - 33.3\% in Class 1 BMI and $42.8 \%$ in Class $2 / 3$ BMI. ${ }^{11}$
In our study, failure of induction was seen in $36.4 \%$ of Class 1 BMI cases and $50 \%$ of Class 2/3 BMI cases. Also, $7.1 \%$ of Class 1 obese pregnant women and $9.1 \%$ of Class 2/3 BMI cases experience prolonged duration of labour (Table 10).

Our study showed increasing incidence of non-progress of labour with increase in BMI - $21.4 \%$ in Class $1 \mathrm{BMI}$ and $36.4 \%$ in Class 2/3 BMI cases (Table 10).

Table 11: Association between BMI classes and intra operative complications.

\begin{tabular}{|c|c|c|c|c|}
\hline \multirow{3}{*}{$\begin{array}{l}\text { Intra - operative } \\
\text { complications }\end{array}$} & \multicolumn{4}{|c|}{ BMI class } \\
\hline & \multicolumn{2}{|c|}{ Class $1(N=15)$} & \multicolumn{2}{|c|}{ Class $2 / 3(N=16)$} \\
\hline & & $\%$ & No & $\%$ \\
\hline $\begin{array}{l}\text { Increased operating time and increased } \\
\text { duration of anaesthesia requirement }\end{array}$ & 03 & 20.0 & 09 & 56.3 \\
\hline
\end{tabular}

Table 12: Association between BMI classes and puerperal complications.

\begin{tabular}{|c|c|c|c|c|}
\hline \multirow{3}{*}{ Puerperal complications } & \multicolumn{4}{|c|}{ BMI class } \\
\hline & \multicolumn{2}{|c|}{ Class $1(N=15)$} & \multicolumn{2}{|c|}{ Class $2 / 3(N=16)$} \\
\hline & No & $\%$ & No & $\%$ \\
\hline Wound discharge & 02 & 13.3 & 03 & 18.8 \\
\hline \multirow[t]{3}{*}{ Wound gape } & 03 & 20.0 & 02 & 12.5 \\
\hline & \multicolumn{2}{|c|}{ Class $1(N=26)$} & \multicolumn{2}{|c|}{ Class $2 / 3(N=21)$} \\
\hline & No & $\%$ & No & $\%$ \\
\hline Lactation failure & 01 & 03.8 & 02 & 09.5 \\
\hline
\end{tabular}

VBAC failure was observed in $60 \%$ of Class $1 \mathrm{BMI}$ cases and $100 \%$ of Class $2 / 3$ BMI cases probably due to a very small sample size $(n=5)$ in our study. The overall incidence of shoulder dystocia in our study was $18.2 \%$ (Table 10).

In our study, post-partum haemorrhage was seen in $11.5 \%$ of Class 1 BMI cases and $23.8 \%$ of Class $2 / 3$ BMI cases (Table 10).

Our study showed an increase in the operating time required for obese pregnant women during caesarean sections and although the dose requirement of the anaesthetic agents is less for obese pregnant women, the duration for which anaesthesia is required increases significantly due to prolonged operating time (Table 11). A study showed that obese pregnant women have an increased risk of dysfunctional labour and caesarean section delivery as discussed above, which are associated with increased requirements for anaesthesia. However, they are also at higher risk of anaesthesia-related morbidity. ${ }^{12}$

Our study showed that overall $16 \%$ of obese pregnant women experienced post-operative wound complications - wound discharge / wound gape despite good antibiotic coverage. Wound discharge was seen in $13.3 \%$ of Class 1 BMI cases and $18.8 \%$ of Class 2/3 BMI cases while wound gape was observed in $20 \%$ of Class 1 BMI and $12.5 \%$ of Class 2/3 BMI cases (Table 12).

Failure of lactation was seen in $3.8 \%$ of Class 1 obese pregnant females and $9.5 \%$ of Class $2 / 3$ obese pregnant females in our study (Table 12).

\section{DISCUSSION}

Our study aimed to study the association of obesity with the maternal and perinatal outcome. The outcomes were compared among Class 1 obese (BMI $30 \mathrm{~kg} / \mathrm{m}^{2}-34.99$ $\left.\mathrm{kg} / \mathrm{m}^{2}\right)$ and Class $2 / 3$ obese $\left(B M I=>35 \mathrm{~kg} / \mathrm{m}^{2}\right)$ pregnant women to understand the effects of increase in BMI on pregnancy outcome. The study concluded that obesity has adverse effects on both maternal as well as fetal outcome along with an increased association with medical comorbidities.

The mean age of the study population was 28 years with age ranging from 20 to 38 years and majority of the cases belonged to the age group of $25-29$ years. The average weight gain was around $8-9 \mathrm{~kg}$ in both the BMI classes.

The rate of abortions in our study $-41.4 \%$ of the Class 1 BMI cases and $28.6 \%$ of the Class $2 / 3$ cases was comparable to a study conducted by Lashen et al. ${ }^{5}$ Infertility was observed in the study population - more in 
Class 2/3 BMI compared to Class 1 BMI. A $2 \%$ lower fertility rate for every $\mathrm{kg} / \mathrm{m}^{2}$ increase in BMI was observed which was similar to a study conducted by Steeg et al, showing a $4 \%$ lower fertility rate for every $\mathrm{kg} / \mathrm{m}^{2}$ increase in BMI. ${ }^{13}$

Complications - maternal and fetal - were present in majority of pregnancies in the study population - slightly more in Class 2/3 BMI compared to Class $1 \mathrm{BMI}$.

The study also showed a positive association of obesity with medical co-morbidities like pregnancy induced hypertension, hypertension, gestational diabetes mellitus, Type 2 diabetes mellitus and hypothyroidism. A metaanalysis by Leddy et al showed an increased risk of hypertensive disorders of pregnancy including preeclampsia in obese pregnant women. ${ }^{4}$ Several large population studies have also shown that obese women are two to three times more likely to develop preeclampsia than their leaner counterparts. ${ }^{14}$ Based on meta-analysis of literature by Chu et al, the risk of developing GDM is about four and eight times higher among obese and severely obese women respectively, compared with normal-weight pregnant women. ${ }^{15}$

The study showed a declining trend of successful vaginal delivery with increase in BMI similar to a study published by Leddy et al, and a retrospective study in Missouri by Caughey et al, which showed that chances of successful vaginal delivery decrease progressively with increase in BMI. ${ }^{4,14}$ The study showed a low but similar rate of operative vaginal delivery in both the classes and a high rate of caesarean sections in obese pregnant women similar to the retrospective study by Caughey et al. ${ }^{11}$ Both the studies showed an increase in the rate of caesarean sections with increase in BMI.

The study also showed a positive association of obesity with intra-partum complications like failure of induction, prolonged labour, non-progress of labour, failure of VBAC, shoulder dystocia and post-partum haemorrhage. The complications increased with increase in the classes of obesity. Various factors that influence failure of induction like nulliparity, prior vaginal delivery and high birth weight babies were not taken into account in this study. Hence, the failure rate in our study was higher compared to a study carried out by Wolfe et al, where the above confounding factors were taken into consideration. ${ }^{10}$ Both the studies however showed an increasing trend of induction failures with increase in BMI. Our study showed that overall $8 \%$ of obese pregnant women experience prolonged duration of labour similar to a study by Norman et al, in which obese women have a longer duration and slower progression of the latent phase of labour. ${ }^{16}$ Also, for obese women, the accelerated phase of labour does not begin until $6 \mathrm{~cm}$ of dilation and it takes more time to reach the accelerated phase of labour. Furthermore, this difference increases with increasing obesity class. A review article published by Fitzsimons et al showed a high rate of non-progress of labour in obese pregnant women 12 similar to our study. Our study showed an overall low success rate of $20 \%$ for vaginal birth after caesarean section probably due to a very small sample size $(n=5)$ in our study while a study by Durnwald et al, with a sample size of about 500 obese pregnant women showed it to be 54.6\%. ${ }^{17}$ However, both the studies affirmed a positive association between decreasing success rate for VBAC and increase in BMI. Although fetal macrosomia is a risk factor for shoulder dystocia, the absolute risk of a severe shoulder dystocia associated with permanent impairment or death, remains low. ${ }^{18}$ A study carried out in London highlighting the adverse impact of obesity on intra-partum and perinatal outcome showed a $47 \%$ incidence of mild PPH (500 $1000 \mathrm{ml})$ and $14 \%$ incidence of major PPH (>1000 ml) in obese pregnant women. ${ }^{19}$

The operating time and duration of anaesthesia required was increased in the study population with significantly increased duration of operating time and anaesthesia in Class 2/3 compared to Class 1 cases.

Puerperal complications - wound discharge, wound gapes and lactation failure was observed in a number of the cases in the study population despite good antibiotic coverage. This was quite high (10 times) when compared to a study by Kiran et al, which showed a post-partum wound infection rate of only $1.6 \% .^{20}$ The high rates in our study might have been due to low socio-economic status of the cases, poor nutritional status and the unhygienic conditions of their homes. Another study by Sebire et al showed a post-partum wound infection rate of $1.34 \%$ in the obese population. ${ }^{21}$ Various studies have found a relation between obesity and delayed onset of lactogenesis. ${ }^{16}$

Incidence of macrosomia (birth weight $>4 \mathrm{~kg}$ ) was more in the study population. The NICU admissions observed in the study population were mainly due to maternal comorbidities.

\section{CONCLUSION}

Considering the adverse effects of obesity on pregnancy outcomes, pre-pregnancy counseling should be done for all overweight and obese females planning conception signifying the negative impact of obesity on maternal and fetal outcome.

Funding: No funding sources

Conflict of interest: None declared

Ethical approval: The study was approved by the Institutional Ethics Committee

\section{REFERENCES}

1. Cedergren MI. Maternal morbid obesity and the risk of adverse pregnancy outcome. Obstet Gynecol. 2004;103(2):219-24. 
2. Begum KS, Sachchithanantham K, De Somsubhra S. Maternal obesity and pregnancy outcome. Clin Exp Obstet Gynecol. 2011;38(1):14-20.

3. Seligman LC, Duncan BB, Branchtein L, Miranda Gaio DS, Mengue SS, Schmidt MI. Obesity and gestational weight gain: Cesarean delivery and labor complications. Rev Saude Publica. 2006;40(3):45765 .

4. Leddy MA, Power ML, Schulkin J. The impact of maternal obesity on maternal and fetal health. Rev Obstet Gynecol. 2008;1(4):170-8.

5. Lashen H, Fear K, Sturdee DW. Obesity is associated with increased risk of first trimester and recurrent miscarriage: Matched case-control study. Hum Reprod. 2004;19(7):1644-6.

6. Asim SS, Naeem H. Pregnancy with obesity -a risk factor for PIH. J Liaquat Univ Med Heal Sci. 2010;9(3):125-9.

7. Han C, Li C, Mao J. High body mass index is an indicator of maternal hypothyroidism, hypothyroxinemia, and thyroid-peroxidase antibody positivity during early pregnancy. BioMed Research Int. 2015:Article ID 351831.

8. Halloran DR, Cheng YW, Wall TC, Macones GA, Caughey AB. Effect of maternal weight on postterm delivery. J Perinatol. 2012;32(2):85-90.

9. Cnattingius S, Villamor E, Johansson S, Bonamy AKE, Persson M, Wikström AK, et al. Maternal obesity and risk of preterm delivery. JAMA. 2013;309(22):2362.

10. Wolfe KB, Rossi RA, Warshak CR. The effect of maternal obesity on the rate of failed induction of labor. Am J Obstet Gynecol. 2011;205(2).

11. Marshall NE, Guild C, Cheng YW, Caughey AB, Halloran DR. The effect of maternal body mass index on perinatal outcomes in women with diabetes. Am J Perinatol. 2014;31(3):249-56.

12. Fitzsimons KJ, Modder Mrcog J. Obesity in pregnancy: risks and management. MedSci IA. 2009;52-62.

13. Van Der Steeg JW, Steures P, Eijkemans MJC, Habbema JDF, Hompes PGA, Burggraaff JM, et al.
Obesity affects spontaneous pregnancy chances in subfertile, ovulatory women. Hum Reprod. 2008;23(2):324-8.

14. Baeten JM, Bukusi EA, Lambe M. Pregnancy complications and outcomes among overweight and obese nulliparous women. Am J Public Health. 2001;91(3):436-40.

15. Chu SY, Callaghan WM, Kim SY, Schmid CH, Lau $\mathrm{J}$, England LJ, et al. Maternal obesity and risk of gestational diabetes mellitus. Diabetes Care. 2007;30(8):2070-6.

16. Norman SM, Tuuli MG, Odibo AO, Caughey AB, Roehl KA, Cahill AG. The effects of obesity on the first stage of labor. Obs Gynecol. 2012;120(1):130-5.

17. Durnwald P, Ehrenberg CM, Mercer HM, Brian, Durnwald CP, Ehrenberg HM, et al. The impact of maternal obesity and weight gain on vaginal birth after cesarean section success. Am J Obstet Gynecol. 2004;191:954-57.

18. Arabin B, Stupin JH. Overweight and obesity before, during and after Pregnancy: Part 2: Evidence-based Risk Factors and Interventions. Geburtshilfe Frauenheilkd. 2014;74(7):646-55.

19. Vinayagam D, Chandraharan E. The adverse impact of maternal obesity on intrapartum and perinatal outcomes. ISRN Obstet Gynecol. 2012;2012:939762.

20. Usha Kiran TS, Hemmadi S, Bethel J, Evans J. Outcome of pregnancy in a woman with an increased body mass index. BJOG An Int J Obstet Gynaecol. 2005;112(6):768-72.

21. Sebire N, Jolly M, Harris J, Wadsworth J, Joffe M, Beard R, et al. Maternal obesity and pregnancy outcome: a study of 287,213 pregnancies in London. Int J Obes. 2001;25:1175-82.

Cite this article as: Jain RS, Shirodkar SD.

Associated comorbidities with obesity in pregnancy and its feto-maternal outcome. Int J Reprod Contracept Obstet Gynecol 2019;8:2785-91. 